\title{
Protective Effect of SGK1 in Rat Hippocampal Neurons Subjected to Ischemia Reperfusion
}

\author{
Wei Zhang ${ }^{\mathrm{a}}$ Chuan yun Qian ${ }^{\mathrm{a}}$ Shu qing Lib \\ aDepartment of Emergency Medicine, The First Affiliated Hospital of Kunming Medical University, \\ bTeaching and Research Section of Pathophysiology, Kunming Medical University, Kunming, Yunnan, \\ China
}

\section{Key Words}

Neurons $•$ Hippocampus $・$ Ischemia reperfusion $•$ SGK1 $•$ Apoptosis $•$ Necrosis

\begin{abstract}
Background/Aims: To investigate the protective effect of SGK1 (serum- and glucocorticoidinducible protein kinase 1 ) in rat hippocampal neurons in vitro and in vivo following ischemia reperfusion (I/R). Methods: Isolated rat hippocampal neurons were subjected to $2 \mathrm{~h}$ of oxygen and glucose deprivation (OGD) then returned to normoxic conditions for 10, 30 or 60 min. Cell apoptosis and protein expression of SGK1 were analyzed. To examine SGK1 function, we overexpressed SGK1 in rat hippocampal neurons. Finally we examined the involvement of PI3K/Akt/GSK3 $\beta$ signaling by treating the cells (untransfected or transfected with expression vector encoding SGK1) with the PI3K inhibitor LY294002. Findings were confirmed in vivo in a rat model of middle cerebral artery occlusion.Results: I/R caused a time-dependent increase in apoptosis, both in vitro and in vivo. SGK1 protein levels decreased significantly under the same conditions. Overexpression of SGK1 reduced apoptosis following OGD or I/R compared to cells transfected with empty vector and subjected to the same treatment, or sham-operated animals. Addition of LY294002 revealed that the action of SGK1 in suppressing apoptosis was mediated by the PI3K/Akt/GSK3 $\beta$ pathway. Conclusion: SGK1 plays a protective role in ischemia reperfusion in rat hippocampal neurons, exerting its effects via the PI3K/Akt/GSK3 $\beta$ pathway.

\section{Introduction}

Serum- and glucocorticoid-inducible protein kinase 1 (SGK1) is a member of a family of serine/threonine kinases that shares $45-55 \%$ homology of the catalytic domain with Akt, cAMP-dependent protein kinase, $\mathrm{p7} 0^{\mathrm{S} 6 \mathrm{~K}}$ and protein kinase $\mathrm{C}$ [1]. It was originally identified 
as an early gene upregulated by glucocorticoid treatment of mammary tumor cells [1]. Later this gene was found to be a cell volume-regulated gene, markedly upregulated within $20 \mathrm{~min}$ by osmotic or isotonic cell shrinkage, and further upregulated in the brain by dehydration [2]. SGK1 is under rapid, direct transcriptional control rather than post-translational control as is the case for most kinases [1] and its promoter region contains many potential transcription factor sites which could account for its unusual stimulus-dependent regulation of expression [3]. Transcriptional control of SGK1 expression is exerted by various stimuli including mineralocorticoids, gonadotropins, 1,25-dihydroxyvitaminD ${ }_{3}$, transforming growth factor (TGF)- $\beta$, interleukin (IL)- 6 and a range of other cytokines [2] and regulation has been shown to be rapid, with appearance or disappearance of SGK1 mRNA occurring within 20 minutes [4]. SGK1 is expressed in a wide variety of species and in mammals is expressed in almost all tissues examined [4], but at highly variable levels. Its subcellular localization may be related to cell function, with serum treatment leading to entry of SGK1 to the nucleus, while osmotic shock causes cytosolic localization, and its enzymatic activity is controlled by the PI3-kinase cascade, which hyperphosphorylates and thus activates SGK1 [3]. Thus the availability, activity and localization of SGK1 are all under the control of multiple signal transduction pathways [3].

An interruption in blood flow to the brain (cerebral ischemia) for more than a few minutes results in irreversible brain damage, commonly known as stroke. This is the third most common cause of death in most industrialized countries, with an estimated annual global mortality of 4.7 million, and was ranked second among the causes of death and disability in the United States in 2010 [5]. It is the major cause of serious, long-term disability, resulting in functional limitations for over one million adults. The incidence of transient ischemic attacks (TIAs or 'mini-strokes') has also recently been found to be far more common than previously thought $[6,7]$, and since stroke incidence increases with increasing age, the global increase in the aging population will obviously exacerbate this problem. Neurons have been shown to be highly vulnerable to periods of ischemia, with those of the hippocampus among the most affected $[8,9]$. Neuronal cell death may occur by both necrosis and apoptosis, with necrosis tending to occur soon after ischemic insult and apoptosis taking precedence later [10]. This may present an opportunity for medical intervention if apoptosis could be prevented within the first few hours of an ischemic attack.

To gain insight into the molecular mechanisms involved in neuronal damage following ischemia-reperfusion, we investigated the activation of protein kinases reported to be associated with mediating ischemic protection in the heart and brain. SGK1 has been reported to show increased expression in the rat hippocampus following global ischemia-reperfusion [11], and is also upregulated after neuronal injury [12]. SGK1 is known to be a regulator of

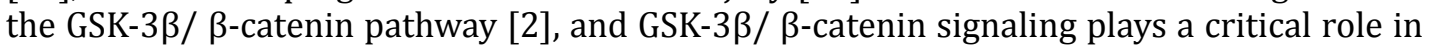
apoptosis of neurons in diseases of the CNS [13]. We used oxygen and glucose deprivation (OGD), an established model of ischemia-reperfusion [14], and examined expression of SGK1 and its effect on cell apoptosis following OGD. Our findings were confirmed in a rat Middle Cerebral Artery Occlusion (MCAO) model of ischemia/reperfusion.

\section{Materials and Methods}

\section{Hippocampal neuronal cell culture}

Experiments were performed according to the international guidelines for animal research, and were designed to use the minimum number of animals and minimize their suffering. All experiments were performed in accordance with the American animal protection legislation and approved by the Institutional Animal Care and Use Committee of the First Affiliated Hospital of Kunming Medical University.

Neonatal Sprague Dawley rat pups (P1) were used to prepare hippocampal neuronal cultures [15]. The hippocampi were dissected in ice-cold dissection solution consisting of $136 \mathrm{mM} \mathrm{NaCl}, 5.4 \mathrm{mM} \mathrm{KCl}, 0.2$ $\mathrm{mM} \mathrm{Na}_{2} \mathrm{HPO}_{4}, 2 \mathrm{mM} \mathrm{KH}_{2} \mathrm{PO}_{4}, 16.7 \mathrm{mM}$ glucose, $20.8 \mathrm{mM}$ sucrose and $10 \mathrm{mM}$ HEPES, pH 7.4, then incubated for $20 \mathrm{~min}$ in a solution of $0.125 \%$ trypsin (Invitrogen, Carlsbad, CA) at $37^{\circ} \mathrm{C}$. At the end of the incubation, 
Dulbecco's modified Eagle's medium (DMEM) (Invitrogen) supplemented with 10\% heat-inactivated fetal bovine serum (FBS) (Hyclone, Logan, UT) and 10\% horse serum (Invitrogen) was added to the hippocampi and a single-cell suspension was produced by mechanically dissociating the cells using a Pasteur pipette with a fire-narrowed tip. Cells were plated into poly-L-lysine (Sigma, St. Louis, MO) coated 6-well plates (Costar, Cambridge, MA), at a density of $1 \times 10^{5} \mathrm{cells} / \mathrm{cm}^{2}$, and incubated in the same medium described above overnight at $37^{\circ} \mathrm{C}$ in $5 \% \mathrm{CO}_{2}$ in air. Next day the cells were changed to serum-free $\mathrm{B} 27 /$ neurobasal medium (Sigma), and maintained for 7 to 10 days, during which half the culture medium was replaced every 3 days.

\section{Immunofluorescence analysis}

Protein expression in vitro: at the end of the culture period, neurons were washed in phosphatebuffered saline (PBS), fixed in $4 \%$ paraformaldehyde for $1 \mathrm{~h}$, then assessed by immunostaining for the neuronspecific marker microtubule-associated protein-2 (MAP-2) and SGK1. Briefly, fixed cells were blocked for 1 $\mathrm{h}$ by incubating with $5 \% \mathrm{BSA}$, then incubated with a mouse monoclonal to MAP-2 or rabbit polyclonal to SGK-1 (both from Abcam), diluted at 1:500 in PBS with 1\% bovine serum albumin (BSA) overnight at $4^{\circ} \mathrm{C}$. After washing three times, the cells were incubated with appropriate secondary antibodies: fluorescein isothiocyanate (FITC)-conjugated anti-mouse IgG (1:100) for MAP-2, and rhodamine-conjugated anti-rabbit IgG $(1: 100)$ for SGK1. Staining was performed for $1 \mathrm{~h}$ in the dark at $37^{\circ} \mathrm{C}$. Samples incubated with nonimmune IgG at the same dilution were used as negative controls. Cell nuclei were counterstained with the DNA-specific dye Hoescht 33258 (Sigma) at a final concentration of $5 \mu \mathrm{g} / \mathrm{mL}$, and specimens were viewed under a Nikon TE2000-S fluorescence microscope (Nikon Inc., Tokyo, Japan).

Protein expression in vivo: Twenty-four hours after reperfusion, rats were anesthetized and perfused with saline, followed by $4 \%$ paraformaldehyde, through the ascending aorta. The brains were then removed and post-fixed in $4 \%$ paraformaldehyde for $24 \mathrm{~h}$, before changing to $20 \%$ sucrose for $2-3$ days, then $30 \%$ sucrose for a further 2-3 days. Brains were then embedded in OCT compound, and $10 \mu \mathrm{m}$ frozen sections were cut for immunostaining. To block non-specific staining, sections were incubated for $2 \mathrm{~h}$ at room temperature in PBS with $10 \%$ non-immune serum, $3 \%(\mathrm{w} / \mathrm{v})$ bovine serum albumin (BSA), $0.1 \%$ Triton $\mathrm{X}-100$ and $0.05 \%$ Tween 20 . The sections were then incubated overnight at $4{ }^{\circ} \mathrm{C}$ with both the antibody against SGK-1 (1:100, Abcam) as above, and a mouse monoclonal antibody against the neuronal marker NeuN (1:1000; Abcam). Next day, sections were washed and incubated for $2 \mathrm{~h}$ at $4^{\circ} \mathrm{C}$ with a mixture of FITCand TRITC-conjugated secondary antibodies to detect neurons and SGK-1, respectively. Control sections were processed without primary antibody. Cells double-labeled for both SGK-1 and NeuN were counted and expressed as a proportion of the NeuN cells; a minimum of $200 \mathrm{NeuN-positive} \mathrm{cells} \mathrm{was} \mathrm{counted} \mathrm{in} 2$ or 3 adjacent sections per rat [16].

\section{Plasmids and transfection}

SGK1 was prepared by amplifying the rat SGK1 mRNA (NM_001193568.1) using the following primers: Forward, 5'-CAAGGAAACGTCAGTGCTCG-3',

Reverse, 5'-ACATGGGAAATACGCAAGGGT-3'

The polymerase chain reaction (PCR) products were subcloned between KpnI and XbaI sites of the mammalian expression vector pCMV4 (BD Biosciences Clontech, Palo Alto, CA) to produce the construct pCMV4-SGK1. All constructs were confirmed by automated DNA sequencing.

Hippocampal neurons were transfected with the empty vector pCMV4 or pCMV4-SGK1 using Lipofectamine 2000 (Invitrogen). Twenty-four hours after transfection, they were trypsinized and seeded for the various assays.

\section{Ischemia reperfusion cell model}

Oxygen and glucose deprivation (OGD) was used as a model of in vivo ischemia conditions [14]. OGD of hippocampal neurons was induced by washing the cells with PBS supplemented with $0.5 \mathrm{mM} \mathrm{CaCl}_{2}$ and 1 $\mathrm{mM} \mathrm{MgCl} 2$ then incubating them in an anaerobic chamber $\left(85 \% \mathrm{~N}_{2}, 5 \% \mathrm{H}_{2^{\prime}}, 10 \% \mathrm{CO}_{2^{\prime}}, 35^{\circ} \mathrm{C}\right.$; Forma Scientific, Marjetta, $\mathrm{OH}$ ) for $120 \mathrm{~min}$. Anaerobic conditions in the chamber were monitored using Gaspack aerobic indicator strips (BD Biosciences, San Jose, CA). Aerobic conditions were restored by removing the cells from the anaerobic chamber, providing fresh medium and returning them to the normal incubator where they were allowed to recover for 10,30 or $60 \mathrm{~min}$. 
To investigate the effect of the PI3K/Akt/GSK3 $\beta$ pathway on the neuroprotective effect exerted by overexpressing SGK1 in the response to OGD, cultures were treated with the PI3K inhibitor LY294002, which was dissolved in $0.2 \%$ dimethyl sulfoxide (DMSO) to a concentration of $10 \mu \mathrm{M}$ and added to cell cultures for $30 \mathrm{~min}$ before subjecting them to OGD.

Flow cytometry analysis

Cell apoptosis was determined by annexin-V/PI double-staining followed by flow cytometric analysis, using an annexin-V-FITC/PI apoptosis detection kit (Sigma). At the end of the culture period, cells were trypsinized, washed twice with cold PBS, then pelleted by centrifugation. The pelleted cells were resuspended in $1 \times$ binding buffer and incubated with staining solution at $4^{\circ} \mathrm{C}$ for 15 min in the dark, then analyzed using a FACSCalibur flow cytometer (BD Biosciences). Ten thousand cells from each sample were scanned and data were analyzed using CellQuest software (BD Biosciences). Necrosis and apoptosis were determined by PI and annexin V-FITC, respectively.

\section{Middle Cerebral Artery Occlusion (MCAO) and Animal Groups}

Male Sprague-Dawley rats weighing 270 to $350 \mathrm{~g}$ were obtained from the Experimental Animals Center, Kunming Medical University, and housed in a facility with a 12-h light/dark cycle at a controlled temperature and humidity with free access to food and water.

The rats were anesthetized with $10 \%$ chloral hydrate $(350 \mathrm{mg} / \mathrm{kg}$, i.p.) and subjected to 2 hours of reversible MCAO, using the intraluminal filament technique [17-20], followed by 1, 6, 12, or $24 \mathrm{~h}$ of reperfusion. Temporalis temperatures were monitored and maintained at $37^{\circ} \mathrm{C} \pm 0.5^{\circ} \mathrm{C}$ using heat lamps and water pads, and a femoral artery catheter was positioned to monitor mean artery blood pressure and arterial blood $\mathrm{pH}$ and $\mathrm{PaCO}_{2}$. Vessel occlusion and reperfusion was assessed by laser-Doppler flowmetry (Moor Instruments, Oxford, UK). Sham-operated group rats underwent the same surgical procedure without MCAO.

Animals were randomly divided into the following groups for treatment:

- $\quad \operatorname{sham}(\mathrm{n}=8)$;

- $\quad$ ischemia/reperfusion (I/R) groups - rats underwent MCAO for 2 hours followed by 1, 6, 12, or 24 $\mathrm{h}$ of reperfusion (four groups, $\mathrm{n}=8$ for each group);

SGK1 group (SGK1, $\mathrm{n}=8$ ) rats were treated with $5 \mu \mathrm{L}$ pCMV4-SGK1 lentivirus plasmid $\left(10^{9}\right.$ transfecting units $/ \mathrm{mL}$ ) via intracerebroventricular (icv) injection 2 weeks before MCAO, and injection of 3\% DMSO (10 $\mu \mathrm{L}$, icv) $15 \mathrm{~min}$ before MCAO, then underwent MCAO for $2 \mathrm{~h}$ followed by $24 \mathrm{~h}$ of reperfusion [21];

$\mathrm{I} / \mathrm{R}$ control group (I/R, $\mathrm{n}=8)$ : rats were injected with $5 \mu \mathrm{L}$ pCMV4 lentivirus plasmids (icv) 2 weeks before MCAO, and injection of 3\% DMSO (10 $\mu \mathrm{L}$, icv) 15 min before MCAO, then underwent MCAO for 2 hours followed by $24 \mathrm{~h}$ of reperfusion.

LY294002 group (LY, $n=8$ ): rats were injected with $5 \mu \mathrm{L}$ pCMV4 lentivirus plasmids (icv) 2 weeks before MCAO [22], and injection of the phosphoinositide 3-kinase inhibitor LY294002 (10 $\mu \mathrm{L}, 10 \mathrm{mM}$, in 3\% DMSO, icv) 15 min before MCAO [23]. They were then subjected to MCAO for $2 \mathrm{~h}$ followed by $24 \mathrm{~h}$ of reperfusion.

SGK1 with LY294002 group (SGK1+LY, $\mathrm{n}=8$ ): rats were injected with $5 \mu \mathrm{L}$ pCMV4-SGK1 lentivirus plasmids $\left(10^{9}\right.$ transducing units/mL, icv $) 2$ weeks before MCAO, and injection of LY294002 $(10 \mu \mathrm{L}, 10 \mathrm{mM}$, in 3\% DMSO, icv) 15 min before MCAO. They were then subjected to MCAO for $2 \mathrm{~h}$ followed by $24 \mathrm{~h}$ of reperfusion.

For plasmid injection, the rats were anesthetized and placed in a stereotaxic apparatus (David Kopf Instruments, Tujunga, CA) then injected into the right lateral ventricle over a 3-min duration with $5 \mu \mathrm{L}$ pCMV4-SGK1 lentivirus plasmid, using a Hamilton microsyringe. A stereotaxic instrument was used to guide the needle into position with the coordinates of $0.8 \mathrm{~mm}$ posterior to the bregma, $1.5 \mathrm{~mm}$ lateral to the midline, and $4.5 \mathrm{~mm}$ ventral to the surface of the skull [21].

At the end of treatment, rats in each group were sacrificed by decapitation, and cortical samples of brain tissue were immediately frozen at $-70^{\circ} \mathrm{C}$ or fixed in $4 \%$ paraformaldehyde for further molecular or histologic analysis.

\section{Evaluation of ischemic infarct area}

Twenty-four hours after reperfusion, rats were deeply anesthetized with $10 \%$ chloral hydrate (350 $\mathrm{mg} / \mathrm{kg}$, i.p.) and sacrificed as described above. The brains were quickly removed, five coronal sections of 
$2 \mathrm{~mm}$ thickness were cut, and sections were stained with 2\% 2,3,5-triphenyltetrazolium chloride (TTC) solution for $20 \mathrm{~min}$ at $37^{\circ} \mathrm{C}$ in the dark. Stained sections were photographed, and infarct tissue was identified as unstained areas. The infarct area as a proportion of each hemisphere was measured using an image analysis system [18].

\section{Western blotting analysis}

Cultured cells or tissue samples were homogenized on ice in lysis buffer consisting of $60 \mathrm{mM}$ Tris$\mathrm{HCl}, 2 \%$ sodium dodecyl sulfate (SDS), $10 \%$ sucrose, $2 \mathrm{mM}$ phenyl methyl sulfonyl fluoride (PMSF), $1 \mathrm{mM}$ sodium orthovanadate, and $10 \mu \mathrm{g} / \mathrm{mL}$ aprotinin. The lysates were briefly sonicated then centrifuged at $15,000 \times \mathrm{g}$ at $4^{\circ} \mathrm{C}$ for $5 \mathrm{~min}$, and the supernatant was collected. Protein concentration was determined using the Bradford assay (Bio-Rad, Hercules, CA) with BSA as the standard [15]. Samples were prepared by diluting in sample buffer $(250 \mathrm{mM}$ Tris- $\mathrm{HCl}, 4 \%$ SDS, $10 \%$ glycerol, $2 \% \beta$-mercaptoethanol and $0.002 \%$ bromophenol blue) to give equivalent amounts of protein, then boiling for $5 \mathrm{~min}$. The prepared samples were separated by $10 \%$ SDS-PAGE then electroblotted onto a nitrocellulose membrane (Invitrogen). The membrane was blocked for $2 \mathrm{~h}$ in $5 \%$ skimmed milk powder in Tris-buffered saline containing $0.1 \%$ Tween 20 (TBST) at room temperature, then incubated with primary antibodies against SGK1 (1:500); cleavedcaspase 3 (1:500); phospho-Akt (S473) (1:200); Akt (1:500); phospho-GSK3 $\beta$ (Y216) (1:500); GSK3 $\beta$ (1:500); or $\beta$-actin (1:1000) (all from Abcam) overnight at $4^{\circ} \mathrm{C}$. Next day the membrane was washed three times in TBST, then incubated for $1 \mathrm{~h}$ at room temperature with the HRP-conjugated goat anti-rabbit IgG H\&L/HRP secondary antibody (Abcam) at 1:2000 [24]. Immunoreactive proteins were detected using an enhanced chemiluminescence detection system (Santa Cruz Biotechnology) and visualized using the Sygene Bio image system (Synoptics Ltd., Cambridge, UK). The $\beta$-actin gene was used as the internal standard for quantitative comparison of expression.

\section{Real-time PCR analysis}

Total RNA was extracted from cultured cells or from brain cortex tissue samples using Trizol reagent (Life Technologies, Carlsbad, CA) according to the manufacturer's instructions. RNA (2 $\mu \mathrm{g})$ was reversetranscribed into cDNA using a High-Capacity cDNA synthesis kit (Applied Biosystems, CA) [25]. Quantitative real-time RT-PCR was performed using SYBR Green in a Rotor-Gene 3000 (Corbett Research, Sydney, Australia) using the following primers for SGK1:

Forward, 5'-CTCGCTTCTACGCAGCTGAA-3';

Reverse, 5'-GACGTTGTCCCATTGTGCTC-3'

with GAPDH as internal standard for quantitative comparison. GAPDH primers:

Forward, 5'-GTCGGTGTGAACGGATTTGG-3';

Reverse, 5'-CCCCATTTGATGTTAGCGGG-3'

For relative quantitation of gene expression the comparative Ct (threshold cycle) method was used [26].

\section{TUNEL staining}

At the end of the study period, rats were sacrificed and perfused as described above. The brains were then removed and post-fixed in $4 \%$ paraformaldehyde for $24 \mathrm{~h}$, before embedding in paraffin and cutting into coronal sections of $4 \mu \mathrm{m}$ thickness. TUNEL staining was performed according to the manufacturer's instructions (Roche molecular Biochemicals Inc., Mannheim, Germany). Briefly, sections were pretreated with Proteinase $\mathrm{K}$ and $0.3 \% \mathrm{H}_{2} \mathrm{O}_{2}$, then incubated with terminal deoxynucleotidyl transferase at $37^{\circ} \mathrm{C}$ for 1 $\mathrm{h}$. They were then transferred to peroxidase-conjugated antibody for $30 \mathrm{~min}$, followed by color development with 3' diaminobenzidene (DAB). Cells displaying brown staining within the nucleus were counted as TUNEL positive cells. The number of TUNEL-positive cells was counted in 3 non-overlapping microscopic fields under high-power magnification (200x) by one observer blinded to the group assignment, and results are expressed as percentage of total cells [22].

\section{Statistical analysis}

The data were expressed as mean \pm S.E. from three independent experiments and the statistical significance was tested by Student's t test. A $P$ value $<0.05$ was considered statistically significant. ${ }^{*} P<0.05$; ${ }^{* *} P<0.01$. 
Fig. 1. Immunofluorescence analysis of SGK1 expression in cultured hippocampal neurons. Cultured hippocampal neurons stained with Hoescht (A), and immunostained for MAP2 (B) and SGK1 (C); (D) Hoescht, MAP2 and SGK1 merged. Original magnifications, $\times 200$.
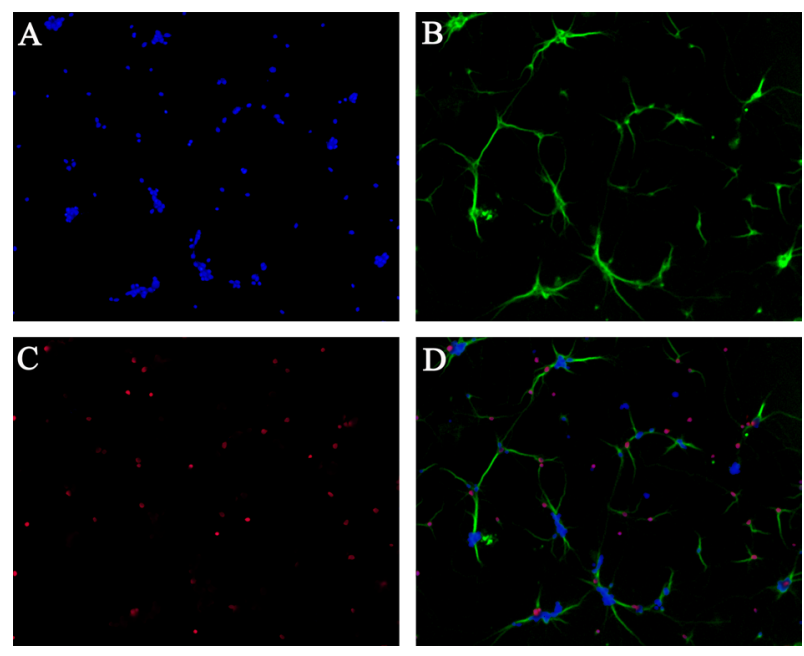

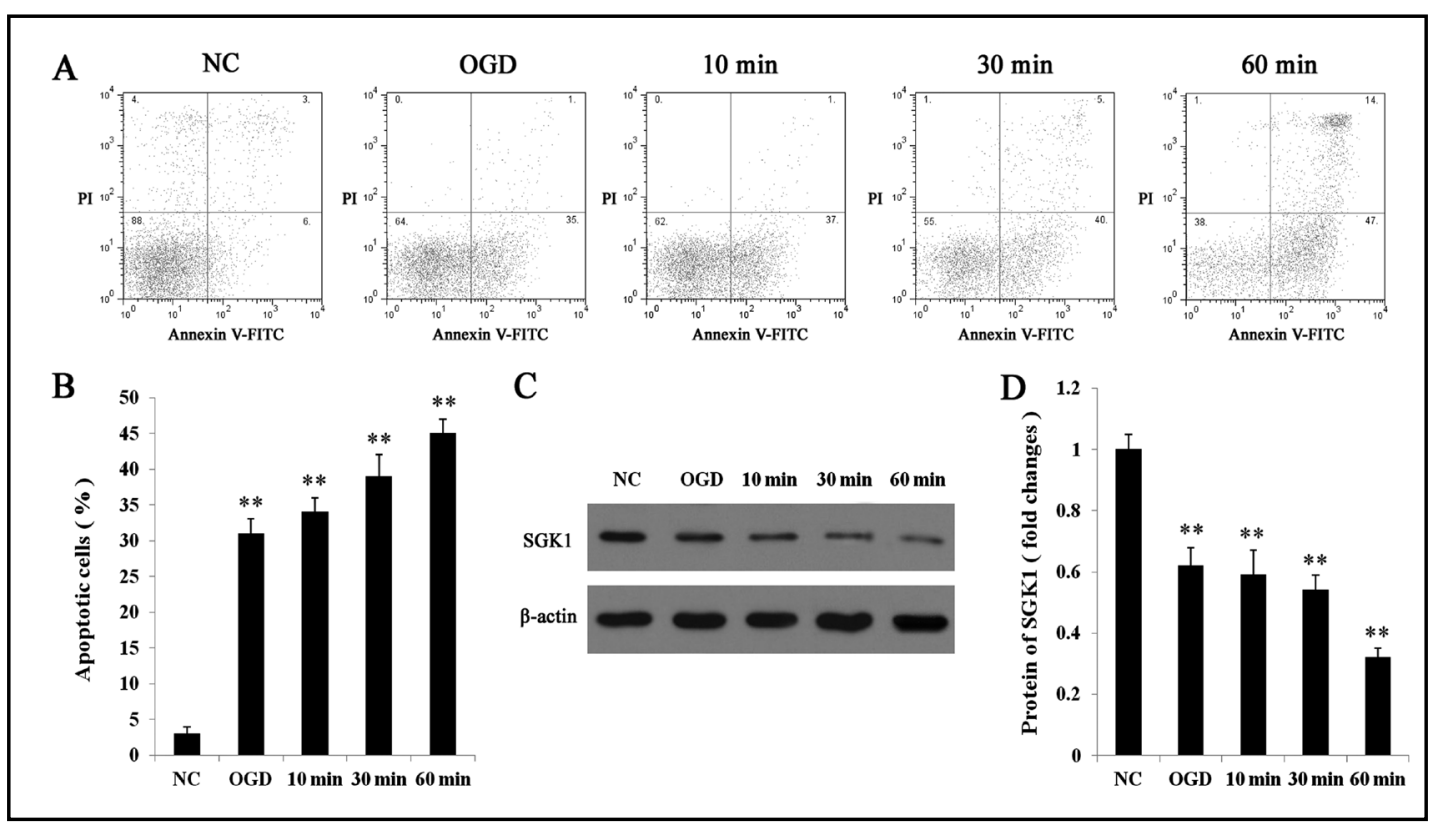

Fig. 2. Flow cytometric analysis of neuronal apoptosis following ischemia-reperfusion. Hippocampal neurons were subjected to $120 \mathrm{~min}$ OGD, and then allowed to recover for 10, 30 or $60 \mathrm{~min}$. Cell apoptosis was determined by flow cytometric analysis with annexin-V/PI double-staining (A-B), while western blotting was used to analyze SGK1 protein levels (C-D). NC, neurons without any treatment; OGD, neurons subjected to $120 \mathrm{~min}$ OGD; $10 \mathrm{~min}$ (30 $\mathrm{min}$ or $60 \mathrm{~min}$ ), cells were allowed to recover for $10 \mathrm{~min}$ (30 $\mathrm{min}$ or $60 \mathrm{~min}$ ) after 120 -min ODG. ${ }^{* *} P<0.01$ vs. NC.

\section{Results}

\section{Expression of SGK1 in cultured hippocampal neurons}

Rat hippocampal neurons were successfully isolated and cultured. As shown in Fig. 1, immunofluorescence analysis revealed that SGK1 was localized mainly in the nucleus of neurons. SGK1-positive cells were also stained by the neuronal marker microtubuleassociated protein 2 (MAP2).

OGD causes apoptosis of hippocampal neurons

Hippocampal neurons were subjected to $120 \mathrm{~min}$ OGD, followed by 10, 30 or $60 \mathrm{~min}$ recovery. Flow cytometric analysis with annexin-V/PI double-staining demonstrated that 


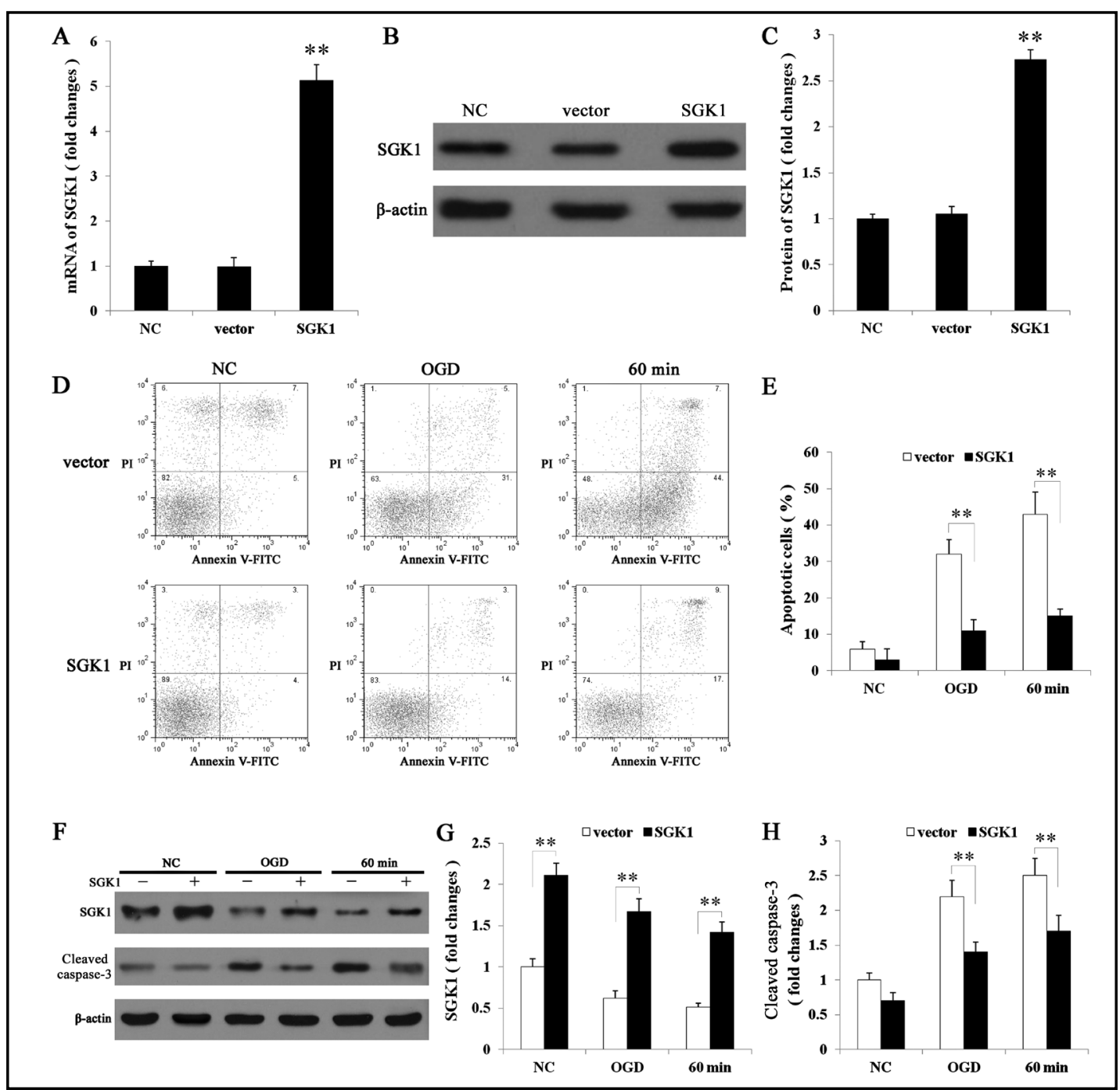

Fig. 3. SGK1-overexpressing neurons are protected from apoptosis following ischemia-reperfusion. Hippocampal neurons were transfected with an empty vector or an expression vector encoding SGK1; $24 \mathrm{~h}$ after transfection, SGK1 mRNA (A) and protein (B-C) levels were determined by real-time PCR and western blot analysis, respectively, ${ }^{* *} P<0.01$ vs. NC. Hippocampal neurons were transfected with an empty vector or an expression vector encoding SGK1 for $24 \mathrm{~h}$, then subjected to $120 \mathrm{~min}$ OGD, and then allowed to recover for $60 \mathrm{~min}$; cell apoptosis was determined by flow cytometric analysis with annexin-V/PI double-staining (DE). SGK1 and activated caspase- 3 protein levels (F-H) were determined by western blot analysis. ${ }^{* *} P<0.01$.

OGD caused increased annexin $V$ staining, which increased with increasing reperfusion time; PI also increased at 30 and 60 min (Fig. 2A). Quantitative analysis of the results revealed that OGD caused a massive increase in apoptosis compared to untreated neuronal cultures; increasing reperfusion time led to further significant, time-dependent increases (Fig. 2B). Western blotting analysis of SGK1 protein levels (Fig. 2C, D) revealed that SGK1 levels were significantly reduced by OGD, and showed further time-dependent decreases with recovery time.

Overexpression of SGK1 blocks apoptosis in hippocampal neurons subjected to OGD

Hippocampal neurons were transfected with an empty vector or an expression vector encoding SGK1, and $24 \mathrm{~h}$ after transfection, SGK1 mRNA (Fig. 3A) and protein (Fig. 3B, C) levels were determined by real-time PCR and western blot analysis, respectively. Transfection 
A
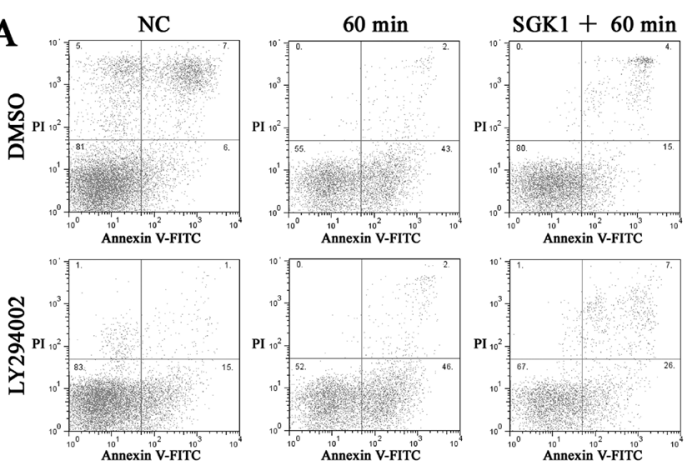

C

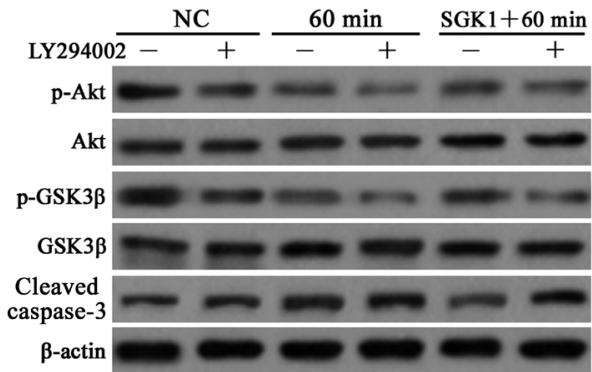

$\mathrm{E}$

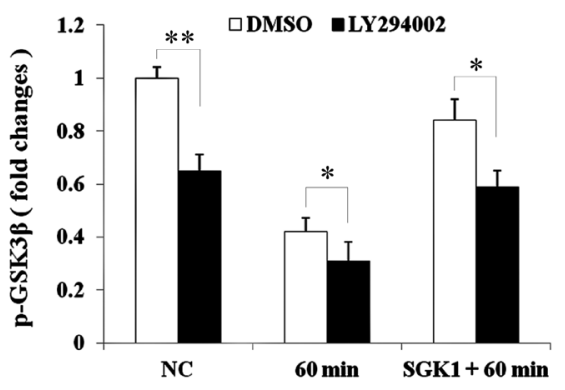

B

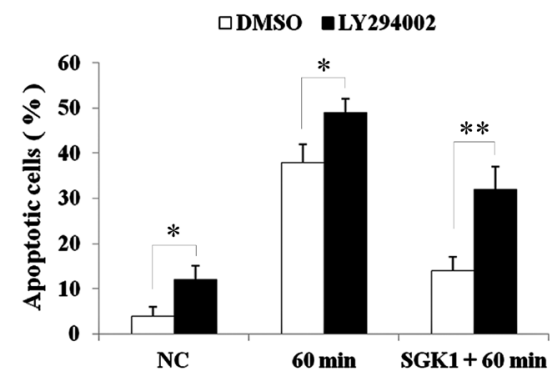

D
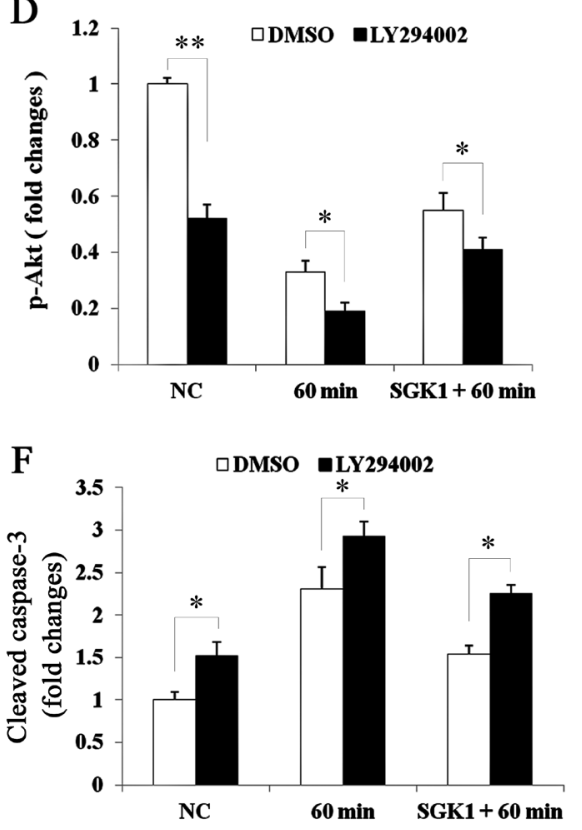

Fig. 4. SGK1 protection is mediated by the PI3K/Akt/GSK3 $\beta$ pathway. Cells (untreated or transfected with an expression vector encoding SGK1 for $24 \mathrm{~h}$ ) were incubated for $30 \mathrm{~min}$ with $10 \mu \mathrm{M}$ LY294002 (or DMSO), then subjected to $120 \mathrm{~min}$ OGD, and then allowed to recover for $60 \mathrm{~min}$; cell apoptosis was determined by flow cytometric analysis with annexin-V/PI double-staining (A-B), p-Akt, Akt, p-GSK3 $\beta$, GSK3 $\beta$, and activated caspase-3 protein levels (C-F) were determined by western blot analysis. ${ }^{*} P<0.05,{ }^{* *} P<0.01$.

with SGK1 resulted in an increase in mRNA of approximately 5 -fold, and an increase of more than 2.5 -fold in protein levels, compared to untreated neurons.

Transfected hippocampal neurons were subjected to 120 min OGD, and then allowed to recover for $60 \mathrm{~min}$, and cell apoptosis was determined by flow cytometric analysis with annexin-V/PI double-staining at the end of the OGD period and after 60 min recovery (Fig. 3D, E). Overexpressing cells subjected to OGD showed a significant reduction in apoptosis compared to vector-transfected neurons, and after $60 \mathrm{~min}$ reperfusion this difference was more marked, with a large increase in apoptosis in control neurons but little effect in the SGK1-overexpressing cells.

Western blot analysis of SGK1 (Fig. 3F, G) protein levels showed a significant decrease in SGK1 expression in OGD-treated versus normal neurons, with a further decrease following $60 \mathrm{~min}$ reperfusion. Although overexpressing cells subjected to OGD then reperfusion showed the same trend, the level of SGK1 expression was significantly increased under all conditions compared to vector controls. 


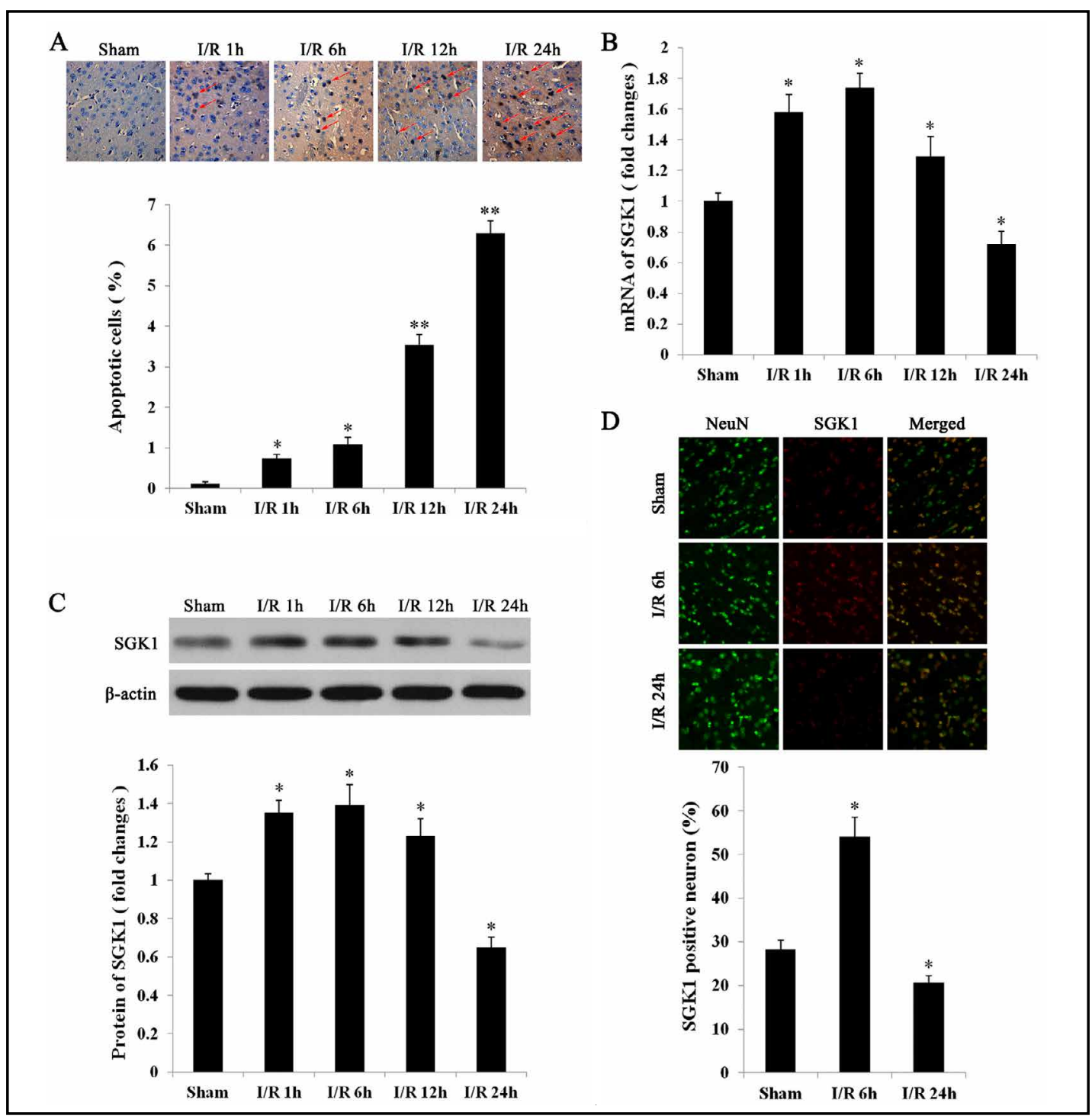

Fig. 5. I/R induces apoptosis in vivo and increases expression of SGK1 in neurons. Rats were subjected to $2 \mathrm{~h}$ of MCAO followed by 1, 6, 12 or $24 \mathrm{~h}$ reperfusion. (A) TUNEL assay was performed on the sections from ischemic penumbral cortex of each group. Magnification 200× (arrows show apoptotic cells) and the percentage of apoptotic cells in the ischemic penumbral cortex of each group was calculated. SGK1 mRNA (B) and protein (C) levels were determined by real-time PCR and western blot analysis, respectively. (D) The localization of SGK1 (red) in neurons (green) was confirmed by double-label immunofluorescent staining with SGK1 and NeuN (a marker of neurons). Magnification 200× and quantitative analysis of NeuN-positive cells expressing SGK1 (\%) in each group was performed. ${ }^{*} P<0.05,{ }^{* *} P<0.01$ vs. Sham-operated group (Sham).

Analysis of expression of cleaved caspase-3 protein revealed the opposite effect (Fig. $3 \mathrm{~F}, \mathrm{H}$ ), with OGD causing caspase-3 expression to more than double in control neurons, while overexpression of SGK1 caused a reduction of cleaved caspase-3 expression in control cultures and a significant reduction in expression in cultures subjected to OGD, either before or after $60 \mathrm{~min}$ of recovery, although levels remained significantly elevated compared to controls. 


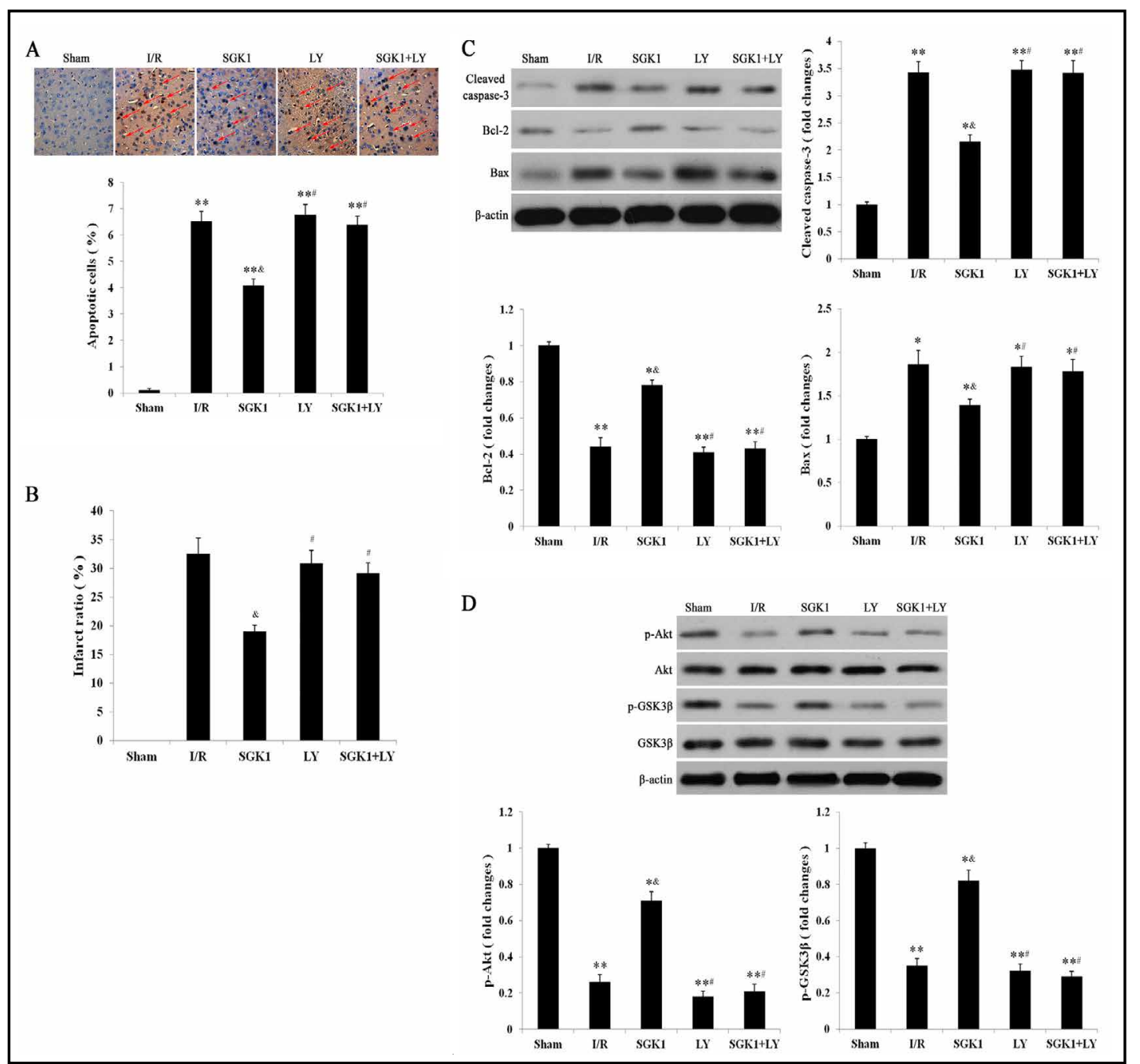

Fig. 6. SGK1 transfection protects against I/R-induced neuronal apoptosis and brain infarct and this effect is blocked by LY294002. Rats were injected with $5 \mu \mathrm{L}$ pCMV4-SGK1 (or pCMV4) lentivirus plasmids 2 weeks before MCAO, and injection of LY294002 (or DMSO) 15 min before MCAO. They were then subjected to MCAO for 2 hours followed by $24 \mathrm{~h}$ of reperfusion. (A) Apoptotic cells were examined by TUNEL assay, Magnification 200× (arrows show apoptotic cells) and percentage of apoptotic cells in the ischemic penumbral cortex of each group was calculated. (B) Infarct size was detected by 2,3,5-triphenyl-2H-tetrazolium chloride (TTC) staining $24 \mathrm{~h}$ after reperfusion, and infarct volume was quantified. Cleaved caspase-3, Bcl-2, Bax (C) and p-Akt, Akt, p-GSK3 $\beta$, GSK3 $\beta$ (D) protein levels were determined by western blot analysis. $* P<0.05$, ${ }^{* *} P<0.01$ vs. Sham-operated group (Sham); ${ }^{\circledR} P<0.05$ vs. I/R group; ${ }^{\sharp} P<0.05$ vs. SGK1 group.

\section{Suppression of apoptosis by SGK1 is mediated by the PI3K/Akt/GSK3 $\beta$ pathway}

Hippocampal neurons, either untransfected or transfected for $24 \mathrm{~h}$ with an expression vector encoding SGK1, were incubated for 30 min with $10 \mu \mathrm{M}$ LY294002, which blocks PI3K-dependent phosphorylation and kinase activity of Akt, before being subjected to 120 min OGD, and then allowed to recover for $60 \mathrm{~min}$. Cell apoptosis was determined by flow cytometric analysis with annexin-V/PI double-staining. Pre-incubation with LY294002 significantly increased apoptosis both in untreated cells and in normal cells subjected to OGD then $60 \mathrm{~min}$ reperfusion, compared to vehicle (DMSO)-treated cells under the same conditions (Fig 4A, B). LY294002 pretreatment caused a larger increase in apoptosis in SGK1-overexpressing cells than in corresponding normal overexpressing cells (Fig. 4B). 
Protein levels of p-Akt, Akt, p-GSK3 $\beta$, GSK3 $\beta$, and activated caspase- 3 were analyzed by western blotting (Fig. 4C-F). Levels of p-Akt were reduced by OGD followed by $60 \mathrm{~min}$ reperfusion and this reduction was suppressed by SGK1 overexpression. In all treatment groups p-Akt was reduced by LY294002 preincubation, although the reduction was greater in untreated controls. Protein levels of p-GSK3 $\beta$ showed a similar trend. In contrast, cleaved caspase- 3 expression was increased more than 2-fold by OGD followed by $60 \mathrm{~min}$ reperfusion, and was suppressed almost to normal levels by SGK1 overexpression. LY294002 preincubation caused a modest, though significant, increase in cleaved caspase- 3 expression in all conditions (Fig. 4C, F).

Ischemia-reperfusion $(I / R)$ induces apoptosis in the intact rat brain, and alters SGK1 expression

Rats were subjected to $2 \mathrm{~h}$ of MCAO followed by reperfusion for $1,6,12$ or $24 \mathrm{~h}$, then TUNEL staining was performed on sections from ischemic penumbral cortex of each group. TUNEL staining was barely observed in the sham-operated brains, while following I/R, the number of TUNEL-positive cells clearly increased with time following reperfusion, reaching more than $6 \%$ at $24 \mathrm{~h}$ (Fig. $5 \mathrm{~A}$ ).

Evaluation of both mRNA (Fig. 5B) and protein (Fig. 5C) showed that SGK1 levels increased within $1 \mathrm{~h}$ after I/R, peaked between 6 and $12 \mathrm{~h}$, and then declined to below control levels. Double immunohistochemical labeling of cells with SGK1 and NeuN, a neuronal marker, confirmed that SGK1 was expressed in rat neurons, and that this expression increased significantly, to almost double the levels in sham-operated animals, by $6 \mathrm{~h}$ after I/R and declined to below control levels after $24 \mathrm{~h} \mathrm{I} / \mathrm{R}$ (Fig. 5D).

SGK1 exerts a protective effect against apoptosis induced by $I / R$, and this effect is blocked by $L Y 294002$

Rats were injected with $5 \mu \mathrm{L}$ pCMV4-SGK1 (or pCMV4) lentivirus plasmids 2 weeks before MCAO, and injection of LY294002 (or DMSO) 15 min before MCAO. They then underwent $2 \mathrm{~h}$ of MCAO followed by reperfusion for $24 \mathrm{~h}$. TUNEL staining of penumbra cortex was barely detectable in the sham-operated brains, but many TUNEL-stained cells were present in the I/R group (Fig. 6A). The number of positive cells was markedly reduced by SGK1 transfection, and this protective effect was blocked by LY294002 treatment. Stained cells were counted and expressed as a percentage of the total cells in the ischemic penumbral cortex of each group, and demonstrated a highly significant increase in apoptotic cells with $\mathrm{I} / \mathrm{R}$ and a significant decrease in this apoptosis with SGK1.

Evaluation of the infarct area by TTC staining revealed large injured areas in rats subjected to I/R, and a significant reduction in infarct area with SGK1 transfection. When these areas were quantified as a proportion of each hemisphere there was clearly a $40 \%$ reduction in the injured area with SGK1 transfection. LY294002 treatment alone had no effect on infarct area, and when combined with SGK1 transfection the protective effect of SGK1 was blocked (Fig. 6B).

Western blotting of tissue lysates showed that I/R induced an increase of more than 3 -fold in protein expression of cleaved caspase 3 and almost 2-fold in the pro-apoptotic protein Bax, while it reduced levels of the anti-apoptotic protein Bcl-2 to less than half. All these changes were partially but significantly reversed by SGK1 transfection (Fig. 6C). In agreement with the observations of infarct area, LY294002 treatment completely blocked the effect of SGK1 on all three indicators (Fig. 6C). Similarly, I/R reduced levels of phosphoAkt and phospho-GSK3 $\beta$ to $26 \%$ and 35\%, respectively, of levels in sham controls, but levels were partially restored by SGK1 transfection to $71 \%$ and $82 \%$, respectively (Fig. 6D).

\section{Discussion}

Hippocampal neurons are particularly vulnerable to death following periods of ischemia [8], and this has been shown to occur by apoptosis both in experimental animals [27-30] 
and in humans [31,32]. Neuronal apoptosis is important both in brain development and in response to insult, and is known to be regulated in a complex manner by a network of interacting pathways, of which the PI3K pathway may be one of the most significant [33]. There is a wealth of evidence demonstrating the importance of the PI3K-Akt signaling pathway in mediating survival signals in neuronal cells [34-36]. The protein kinase SGK, which is related to Akt and also activated by PI3K, has also been reported to be involved in mediating survival signals, acting in other cell types via phosphorylation of the forkhead transcription factor FKHRL1 [37]. It has also been shown to regulate the GSK-3 $\beta / \beta$-catenin signaling pathway in neurons [16].

Glycogen synthase kinase (GSK)-3 $\beta$ was originally identified for its role in energy metabolism, but is now recognized as a multifunctional kinase involved in many different cellular functions including regulation of cell survival under a variety of different conditions [13]. There is evidence that GSK-3 $\beta$ is important in modulating cell responses in the central nervous system $[16,38]$. The role of GSK3 activation in the regulation of apoptosis remains controversial however, with some studies showing that it promotes apoptosis [39] while others suggest it has a protective effect [40].

In this study we isolated rat hippocampal neurons and confirmed that they express SGK1. Using OGD as a model of ischemia reperfusion, we found that apoptosis was greatly increased by OGD as expected, and that it also continued to increase with increasing reperfusion time. Expression of SGK1 followed an inverse profile, decreasing with OGD and continuing to decrease over time.

To investigate whether the reduction in SGK1 is involved in the induction of apoptosis, we used a transfection vector to overexpress SGK1 in our cultured neurons. Overexpression of SGK1 was able to markedly reduce apoptosis induced by OGD, demonstrated both by FACS analysis and by a significant reduction in caspase-3 expression. We then investigated the activation of other protein kinases which reportedly mediate ischemic protection, and found that OGD followed by $60 \mathrm{~min}$ reperfusion suppresses phosphorylation of both Akt and GSK-3 $\beta$ while increasing caspase-3 expression. Overexpression of SGK1 was able to at least partially restore activation of Akt and GSK-3 $\beta$. We used the inhibitor LY294002 to investigate the involvement of the PI3K-Akt signaling pathway in protein phosphorylation and the induction of apoptosis. Our results show that SGK1 suppression of apoptosis in hippocampal neurons is mediated, at least partly, via the PI3K/Akt signaling pathway, and suggest that both Akt and GSK-3 $\beta$ have a protective role in hippocampal neurons exposed to ischemiareperfusion.

To confirm that these results are relevant to the in vivo situation, adult male rats were subjected to MCAO as a model of I/R. When MCAO was followed by reperfusion for between 1 and $24 \mathrm{~h}$, TUNEL staining clearly showed a time-dependent increase in apoptosis following I/R. Over the same time-course, analysis of mRNA and protein expression revealed that SGK1 levels transiently increased before returning to control levels by 24 $\mathrm{h}$, while immunohistochemistry confirmed that the SGK1-positive cells were neurons. These observations suggested that increased SGK1 expression by neurons may occur as a protective response following I/R. We then investigated whether SGK1 does in fact exert a protective effect in vivo. Rats were transfected with the SGK1-expressing plasmid 2 weeks before MCAO and reperfusion. The results showed that the marked increase in apoptosis observed following I/R was markedly reduced by SGK1 transfection. This was reflected in changes in the expression of pro- and anti-apoptotic proteins evaluated by western blotting; altered protein expression patterns induced by I/R were found to be partially reversed by SGK1 transfection. Using TTC staining, we evaluated the effects of I/R on whole rat brains, with or without SGK1 transfection, and confirmed that the large infarct area induced by I/R was significantly reduced by SGK1.

Our in vitro results suggested that the protective effect of SGK1 is mediated, at least partly, via the PI3K/Akt signaling pathway so to test this in vivo we treated the plasmidtransfected rats with LY294002 shortly before MCAO. Similar to the in vitro results, we found that the effect of SGK1 in protecting neurons against apoptosis was blocked by treatment 
Zhang/Qian/Li: SGK1 Protects Neurons from Cell Death

with LY294002, confirming the involvement of the PI3K/Akt signaling pathway in this protective mechanism.

Our results provide new insight into the mechanisms of apoptosis as a result of cerebral ischemic injury and suggest that SGK1 has the potential to protect neurons from these events. This raises the exciting possibility that anti-apoptotic treatment could substantially reduce brain trauma in patients if administered within a few hours of an ischemic attack. Further research will be necessary to reveal whether such an intervention could be feasible in the clinic.

\section{Acknowledgements}

This study was supported by grants from the National Natural Science Foundation of China (NO. 30971171), National Key Technology R\&D Program (2014BAIO1B00), the Education Department of Yunnan province (NO. 2013Y281), and the Dr. Innovation of Kunming Medical University (NO. 2012D12).

\section{References}

1 Webster MK, Goya L, Ge Y, Maiyar AC, Firestone GL: Characterization of sgk, a novel member of the serine/ threonine protein kinase gene family which is transcriptionally induced by glucocorticoids and serum. Mol Cell Biol 1993;13:2031-2040.

-2 Lang F, Bohmer C, Palmada M, Seebohm G, Strutz-Seebohm N, Vallon V: (patho)physiological significance of the serum- and glucocorticoid-inducible kinase isoforms. Physiol Rev 2006;86:1151-1178.

-3 Firestone GL, Giampaolo JR, O'Keeffe BA: Stimulus-dependent regulation of serum and glucocorticoid inducible protein kinase (sgk) transcription, subcellular localization and enzymatic activity. Cell Physiol Biochem 2003;13:1-12.

4 Waldegger S, Barth P, Raber G, Lang F: Cloning and characterization of a putative human serine/threonine protein kinase transcriptionally modified during anisotonic and isotonic alterations of cell volume. Proc Natl Acad Sci U S A 1997;94:4440-4445.

5 Murray CJ, Lopez AD: Measuring the global burden of disease. N Engl J Med 2013;369:448-457.

6 Johnston SC, Fayad PB, Gorelick PB, Hanley DF, Shwayder P, van Husen D, Weiskopf T: Prevalence and knowledge of transient ischemic attack among us adults. Neurology 2003;60:1429-1434.

7 Sonni S, Thaler DE: Transient ischemic attack: Omen and opportunity. Cleve Clin J Med 2013;80:566-576.

-8 Kirino T: Delayed neuronal death in the gerbil hippocampus following ischemia. Brain Res 1982;239:57-69.

-9 Dirnagl U, Iadecola C, Moskowitz MA: Pathobiology of ischaemic stroke: An integrated view. Trends Neurosci 1999;22:391-397.

10 Chen J, Nagayama T, Jin K, Stetler RA, Zhu RL, Graham SH, Simon RP: Induction of caspase-3-like protease may mediate delayed neuronal death in the hippocampus after transient cerebral ischemia. J Neurosci 1998;18:4914-4928.

11 Nishida Y, Nagata T, Takahashi Y, Sugahara-Kobayashi M, Murata A, Asai S: Alteration of serum/ glucocorticoid regulated kinase-1 (sgk-1) gene expression in rat hippocampus after transient global ischemia. Brain Res Mol Brain Res 2004;123:121-125.

$>12$ Imaizumi K, Tsuda M, Wanaka A, Tohyama M, Takagi T: Differential expression of sgk mrna, a member of the ser/thr protein kinase gene family, in rat brain after cns injury. Brain Res Mol Brain Res 1994;26:189196.

13 Jacobs KM, Bhave SR, Ferraro DJ, Jaboin JJ, Hallahan DE, Thotala D: Gsk-3beta: A bifunctional role in cell death pathways. Int J Cell Biol 2012;2012:930710.

14 Pignataro G, Meller R, Inoue K, Ordonez AN, Ashley MD, Xiong Z, Gala R, Simon RP: In vivo and in vitro characterization of a novel neuroprotective strategy for stroke: Ischemic postconditioning. J Cereb Blood Flow Metab 2008;28:232-241.

15 Sheng H, Xu Y, Chen Y, Zhang Y, Ni X: Corticotropin-releasing hormone stimulates mitotic kinesin-like protein 1 expression via a plc/pkc-dependent signaling pathway in hippocampal neurons. Mol Cell Endocrinol 2012;362:157-164.

16 Wu X, Mao H, Liu J, Xu J, Cao J, Gu X, Cui G: Dynamic change of sgk expression and its role in neuron apoptosis after traumatic brain injury. Int J Clin Exp Pathol 2013;6:1282-1293.

17 Cheng J, Uchida M, Zhang W, Grafe MR, Herson PS, Hurn PD: Role of salt-induced kinase 1 in androgen neuroprotection against cerebral ischemia. J Cereb Blood Flow Metab 2011;31:339-350. 
18 Sun B, Chen L, Wei X, Xiang Y, Liu X, Zhang X: The akt/gsk-3beta pathway mediates flurbiprofen-induced neuroprotection against focal cerebral ischemia/reperfusion injury in rats. Biochem Biophys Res Commun 2011;409:808-813.

19 Cheng J, Alkayed NJ, Hurn PD: Deleterious effects of dihydrotestosterone on cerebral ischemic injury. J Cereb Blood Flow Metab 2007;27:1553-1562.

20 Uchida M, Palmateer JM, Herson PS, DeVries AC, Cheng J, Hurn PD: Dose-dependent effects of androgens on outcome after focal cerebral ischemia in adult male mice. J Cereb Blood Flow Metab 2009;29:1454-1462.

-21 Hu Q, Chen C, Khatibi NH, Li L, Yang L, Wang K, Han J, Duan W, Zhang JH, Zhou C: Lentivirus-mediated transfer of mmp-9 shrna provides neuroprotection following focal ischemic brain injury in rats. Brain Res 2011;1367:347-359.

22 Yuan Y, Guo Q Ye Z, Pingping X, Wang N, Song Z: Ischemic postconditioning protects brain from ischemia/ reperfusion injury by attenuating endoplasmic reticulum stress-induced apoptosis through pi3k-akt pathway. Brain Res 2011;1367:85-93.

23 Peng B, Guo QL, He ZJ, Ye Z, Yuan YJ, Wang N, Zhou J: Remote ischemic postconditioning protects the brain from global cerebral ischemia/reperfusion injury by up-regulating endothelial nitric oxide synthase through the pi3k/akt pathway. Brain Res 2012;1445:92-102.

-24 Salma J, McDermott JC: Suppression of a mef2-klf6 survival pathway by pka signaling promotes apoptosis in embryonic hippocampal neurons. J Neurosci 2012;32:2790-2803.

25 Andres-Mateos E, Brinkmeier H, Burks TN, Mejias R, Files DC, Steinberger M, Soleimani A, Marx R, Simmers JL, Lin B, Finanger Hedderick E, Marr TG, Lin BM, Hourde C, Leinwand LA, Kuhl D, Foller M, Vogelsang S, Hernandez-Diaz I, Vaughan DK, Alvarez de la Rosa D, Lang F, Cohn RD: Activation of serum/glucocorticoidinduced kinase 1 (sgk1) is important to maintain skeletal muscle homeostasis and prevent atrophy. EMBO Mol Med 2013;5:80-91.

26 Livak KJ, Schmittgen TD: Analysis of relative gene expression data using real-time quantitative pcr and the 2(-delta delta c(t)) method. Methods 2001;25:402-408.

27 Choi DW: Ischemia-induced neuronal apoptosis. Curr Opin Neurobiol 1996;6:667-672.

28 Hokari M, Kuroda S, Kinugawa S, Ide T, Tsutsui H, Iwasaki Y: Overexpression of mitochondrial transcription factor a (tfam) ameliorates delayed neuronal death due to transient forebrain ischemia in mice. Neuropathology 2010;30:401-407.

29 Itoh T, Satou T, Nishida S, Tsubaki M, Imano M, Hashimoto S, Ito H: Edaravone protects against apoptotic neuronal cell death and improves cerebral function after traumatic brain injury in rats. Neurochem Res 2010;35:348-355.

-30 Nitatori T, Sato N, Waguri S, Karasawa Y, Araki H, Shibanai K, Kominami E, Uchiyama Y: Delayed neuronal death in the ca1 pyramidal cell layer of the gerbil hippocampus following transient ischemia is apoptosis. J Neurosci 1995;15:1001-1011.

-31 Petito CK, Feldmann E, Pulsinelli WA, Plum F: Delayed hippocampal damage in humans following cardiorespiratory arrest. Neurology 1987;37:1281-1286.

32 Macas J, Nern C, Plate KH, Momma S: Increased generation of neuronal progenitors after ischemic injury in the aged adult human forebrain. J Neurosci 2006;26:13114-13119.

-33 Brunet A, Datta SR, Greenberg ME: Transcription-dependent and -independent control of neuronal survival by the pi3k-akt signaling pathway. Curr Opin Neurobiol 2001;11:297-305.

34 Yao R, Cooper GM: Requirement for phosphatidylinositol-3 kinase in the prevention of apoptosis by nerve growth factor. Science 1995;267:2003-2006.

-35 Eves EM, Xiong W, Bellacosa A, Kennedy SG, Tsichlis PN, Rosner MR, Hay N: Akt, a target of phosphatidylinositol 3-kinase, inhibits apoptosis in a differentiating neuronal cell line. Mol Cell Biol 1998;18:2143-2152.

-36 Crowder RJ, Freeman RS: Phosphatidylinositol 3-kinase and akt protein kinase are necessary and sufficient for the survival of nerve growth factor-dependent sympathetic neurons. J Neurosci 1998;18:2933-2943.

37 Brunet A, Park J, Tran H, Hu LS, Hemmings BA, Greenberg ME: Protein kinase sgk mediates survival signals by phosphorylating the forkhead transcription factor fkhrl1 (foxo3a). Mol Cell Biol 2001;21:952-965.

38 Hu S, Begum AN, Jones MR, Oh MS, Beech WK, Beech BH, Yang F, Chen P, Ubeda OJ, Kim PC, Davies P, Ma Q, Cole GM, Frautschy SA: Gsk3 inhibitors show benefits in an alzheimer's disease (ad) model of neurodegeneration but adverse effects in control animals. Neurobiol Dis 2009;33:193-206.

-39 Watcharasit P, Bijur GN, Zmijewski JW, Song L, Zmijewska A, Chen X, Johnson GV, Jope RS: Direct, activating interaction between glycogen synthase kinase-3beta and p53 after DNA damage. Proc Natl Acad Sci U S A 2002;99:7951-7955.

40 Kotliarova S, Pastorino S, Kovell LC, Kotliarov Y, Song H, Zhang W, Bailey R, Maric D, Zenklusen JC, Lee J, Fine HA: Glycogen synthase kinase-3 inhibition induces glioma cell death through c-myc, nuclear factorkappab, and glucose regulation. Cancer Res 2008;68:6643-6651. 